ARTICLE

\title{
New Approach to Alpha Spectrum Analysis: Iterative Monte Carlo Simulations and Fitting
}

\author{
Teemu SIISKONEN* and Roy PÖLLÄNEN \\ STUK - Radiation and Nuclear Safety Authority, PO Box 14, FI-00881 Helsinki, Finland
}

\begin{abstract}
A novel alpha spectrum analysis program which combines the Monte Carlo simulations with spectrum fitting is introduced. The shapes used in the fitting are obtained from the simulations. The program is developed especially to analyze complex alpha particle energy spectra - such as those obtained from non-processed aerosol filters, swipe samples or isolated particles emitting alpha radiation. In addition to activities of the nuclides present in the sample, the program can provide source characterization. In particular, the program can be used to characterize samples of nuclear material, i.e. those containing fissionable isotopes such as ${ }^{235} \mathrm{U}$ or ${ }^{239} \mathrm{Pu}$. In the present paper we illustrate the use of the program to identify and quantify alpha-particle emitting isotopes in a depleted U projectile found in Kosovo and to estimate the depth distribution of alpha-particle emitting aerosols in a glassfibre filter.
\end{abstract}

KEYWORDS: alpha spectrometry, Monte Carlo, non-destructive analysis methods, spectrum analysis

\section{Introduction}

Preventing the proliferation of nuclear materials to unauthorized parties is one of the main security issues the nuclear community faces ${ }^{1,2)}$ - of special interest are the heavy, fissionable isotopes such as ${ }^{235} \mathrm{U}$ and ${ }^{239} \mathrm{Pu}$. They emit alpha radiation of short range, approximately $3.0 \mathrm{~cm}$ and $3.8 \mathrm{~cm}$ in air, respectively. This is one of the reasons why their detection, identification and quantification are challenging. Typically the presence of these isotopes is verified (or excluded) by gamma-ray spectrometry (assuming that the activity is high enough) or by sampling, subsequent radiochemistry and alpha particle counting in a laboratory. The drawback in using radiochemistry is that the sample is destroyed in the process. However, the alpha particle energy spectra can also be measured from unprocessed samples, such as swipes or even from individual radioactive particles. This method, known as non-destructive alpha spectrometry, leaves the sample intact and available for further analyses. The omission of radiochemistry means that the method may easily be used in the field conditions.

The omission of the radiochemistry also means that the samples may be relatively thick (with respect to the range of the alpha radiation) and of irregular shape. The resulting peak broadening (Fig. 1) in the measured spectrum may prevent from using traditional spectrum unfolding programs. Strongly overlapping asymmetric peaks from unprocessed samples call for realistic, usually complex peak shapes. In the approach described in this work these shapes are obtained from the Monte Carlo simulation program called AASI. ${ }^{3)}$ The simulated peak shapes are subsequently used in the peak fitting program known as AASIFIT. Peak shape simulation and the fitting to the measured spectrum are ite-

*Corresponding author, E-mail: teemu.siiskonen@stuk.fi

(C) 2011 Atomic Energy Society of Japan, All Rights Reserved. rated with varying sample properties as long as the fit is deemed acceptable.

The AASIFIT results can be used not only for nuclide identification but also to examine the sample properties such as shape or density. For example, earlier applications include analysis of swipe samples ${ }^{4)}$ and examination of the properties of nuclear bomb particles and particles composed of $U .^{5,6)}$ In this contribution an object made of $U$ (a penetrator found in Kosovo) is used to demonstrate the capabilities of the AASI/AASIFIT program package. Measurements, simulations and spectrum analysis through fitting are used to demonstrate that the program is capable of providing information on the isotopic composition of the projectile. In a case of suspected nuclear material the knowledge of whether the material is processed or not is of utmost importance. Ear-

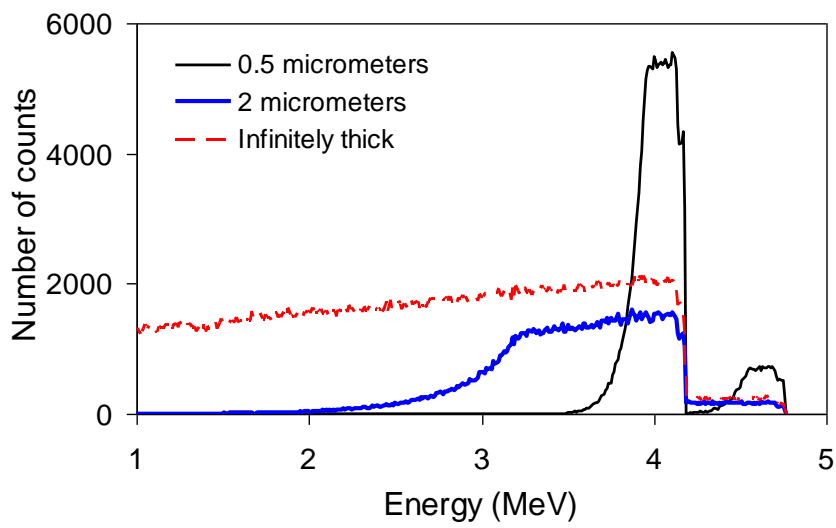

Fig. 1 Simulated spectra from homogeneously distributed alpha emitters in $U$ sources. The simulated nuclides were ${ }^{234} U$ and ${ }^{238} \mathrm{U}$ with an activity ratio of 0.122 (see Section III). Source thicknesses were $0.5 \mu \mathrm{m}$ (thin line), $2 \mu \mathrm{m}$ (thick line) and large compared to the range of alpha particles (dashed line). 
lier, this information was available only after tedious and time-consuming radiochemical processing and counting in a laboratory. In addition, to demonstrate the iterative fitting process, the depth distribution of alpha-particle emitting aerosols in a glassfibre filter is analyzed using AASIFIT.

\section{Simulation and Fitting Programs}

\section{AASI}

Advanced Alpha-Spectrometric Simulation (AASI) is a Monte Carlo program designed for simulations of alpha-particle energy spectra in variety of applications. ${ }^{3)}$ AASI was originally developed for simulations of alpha spectra from aerosol samples, but later the program was extended to deal with other sources emitting alpha radiation. AASI simulates the detection efficiency, detector response and the energy loss of alpha particles in the source and in the materials between the source and the detector. Various types of sources can be used in the computations, including spherical or ellipsoidal radioactive particles, homogeneous thick sources of different shapes and radioactive particles distributed inside a non-active source matrix. Random variations in the source thickness can be accounted for in the simulations. Full decay schemes of the nuclides can be used in the simulations. Emission of gamma rays, characteristic X-rays, conversion electrons and Auger electrons which follow the alpha decay can be also be taken into account in the computations. Thus, cases corresponding to real measurements can be simulated. AASI is validated against other simulation programs and measurements. It has a simple-to-use graphical user interface ${ }^{7)}$ to determine all the essential parameters for the simulation and plotting capabilities for displaying the results. The energy spectra can be imported in different ASCII formats.

The energy loss of the alpha particles in the source and in the material between the source and the active volume of the detector is calculated in continuous energy loss approximation, i.e. the hard collisions leading to large angular deflections are not simulated. The energy loss straggling is estimated by a Gaussian energy distribution. However, the alpha particle scattering in the source backing plate is explicitly simulated with a screened elastic Rutherford scattering model. See Reference 3 for more detailed information about the physics modeling in the program.

\section{AASIFIT}

The output of the AASI program is a histogram representing the energy spectrum registered by the detector. The spectrum consists of pulses from alpha particles and, if included in the simulation, from photons and electrons. AASIFIT uses this histogram as a basis for fitting the simulated data to the measured peaks (so-called area fitting). The peak shape can change from one nuclide to another or even from one alpha line to another. The peaks belonging to one nuclide are fitted together as a group without adjusting the relative areas of the peaks. This results in a robust fit even in a case when multiple nuclides are present in the sample.

The typical fitting process is indicated in Fig. 2. First, the

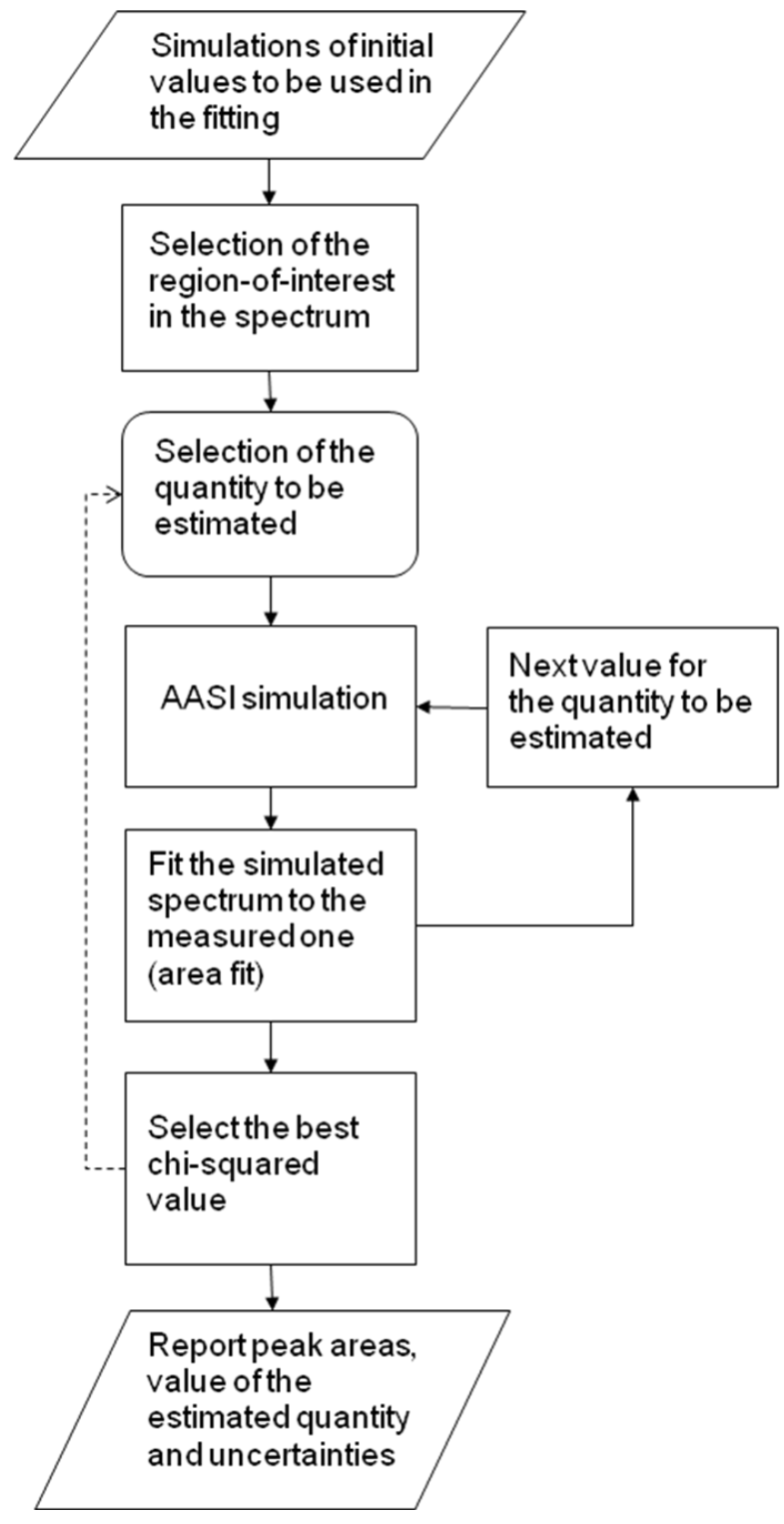

Fig. 2 Flowchart of a typical fitting process.

source properties (e.g. shape, density and nuclide composition) are examined with AASI to get proper initial values for the fitting. Then, AASIFIT can either be used to estimate both the peak areas and one source property or to fit the peak areas only. If multiple source properties need to be estimated through fitting, the fitting process of Fig. 2 can be repeated for each property and, if needed, manually iterated. This is indicated by the dashed line in Fig. 2.

The Levenberg-Marquardt algorithm ${ }^{8,9)}$ is used in the peak area fitting. When the user-selected quantity is deduced from the fit, a simple linear stepping algorithm or a bisection algorithm may be used. After the fitting procedure terminates, AASIFIT reports the peak areas and the value of the user-selected quantity which gave the best chi-squared value. If needed, the nuclide activities can be deduced from the fitted peak areas and the simulated detection efficiency.

The smoothness of the energy histogram of AASI depends on the number of alpha particle emissions simulated: increasing the number of emissions leads to smoother histo- 
gram, longer running times and, in general, better chi-squared values in the fit. Typical running time for a fit is of the order of one minute (assuming that the initial values and the range to be fitted are reasonable). The fitting can easily be carried out on a standard PC.

\section{Examples}

\section{Characterization of a Uranium Projectile}

The object analyzed in this example is a projectile found in Kosovo, in the area where the military troops had used munitions composed of depleted uranium (DU). The shape of the penetrator was cylindrical with a conical front part. The length of the penetrator was $97 \mathrm{~mm}$, the maximum diameter $16.2 \mathrm{~mm}$ and the mass $298.8 \mathrm{~g}$. An earlier investigation $^{10)}$ of the same DU penetrator showed that it is almost exclusively composed of ${ }^{238} \mathrm{U}$, with the mass abundance of $99.79 \%$. Small amounts (in terms of mass) of ${ }^{234} U$, ${ }^{235} \mathrm{U}$ and ${ }^{236} \mathrm{U}$ were also detected.

In this contribution we assume (for the purpose of demonstrating the capabilities of AASIFIT) that the penetrator has an unknown $\mathrm{U}$ composition and use non-destructive alpha spectrometry to analyze the activity ratios of alpha-particle emitting $U$ isotopes in it. Registration of the alpha-particle energy spectrum was done in air, using a semiconductor detector (CAM PIPS, Canberra Inc.). This detector is primarily designed for continuous aerosol monitoring devices and it can be operated at normal air pressure. It also has a light-tight rugged window in front of the active part of the detector, making it suitable for field use. The drawback resulting from the thick entrance window is a lower energy resolution compared to standard detectors designed for alpha-particle counting in a vacuum chamber. However, in non-destructive alpha spectrometry the lower resolution does not generally impede the spectrum analysis since the source characteristics has a major influence on the spectrum quality.

The energy spectrum was measured at $10 \mathrm{~mm}$ distance from the projectile surface. The energy loss of the alpha particles in air between the projectile and the detector shifts the position of the peaks from their nominal positions. In addition, since the penetrator is thick compared to the range of alpha particles in $\mathrm{U}$ (in which the projected range of ${ }^{239} \mathrm{Pu}$ alpha particles is $9 \mu \mathrm{m}$ ), the registered peaks in the spectrum (Fig. 3) have long tails at the low-energy sides. The measurement is a typical example of an alpha spectrum from a thick, homogeneous sample and represents a case where field measurements are done to identify isotopic composition of an unknown macroscopic object. In terms of peak broadening, the spectrum in Fig. 3 is a worst-case scenario out of

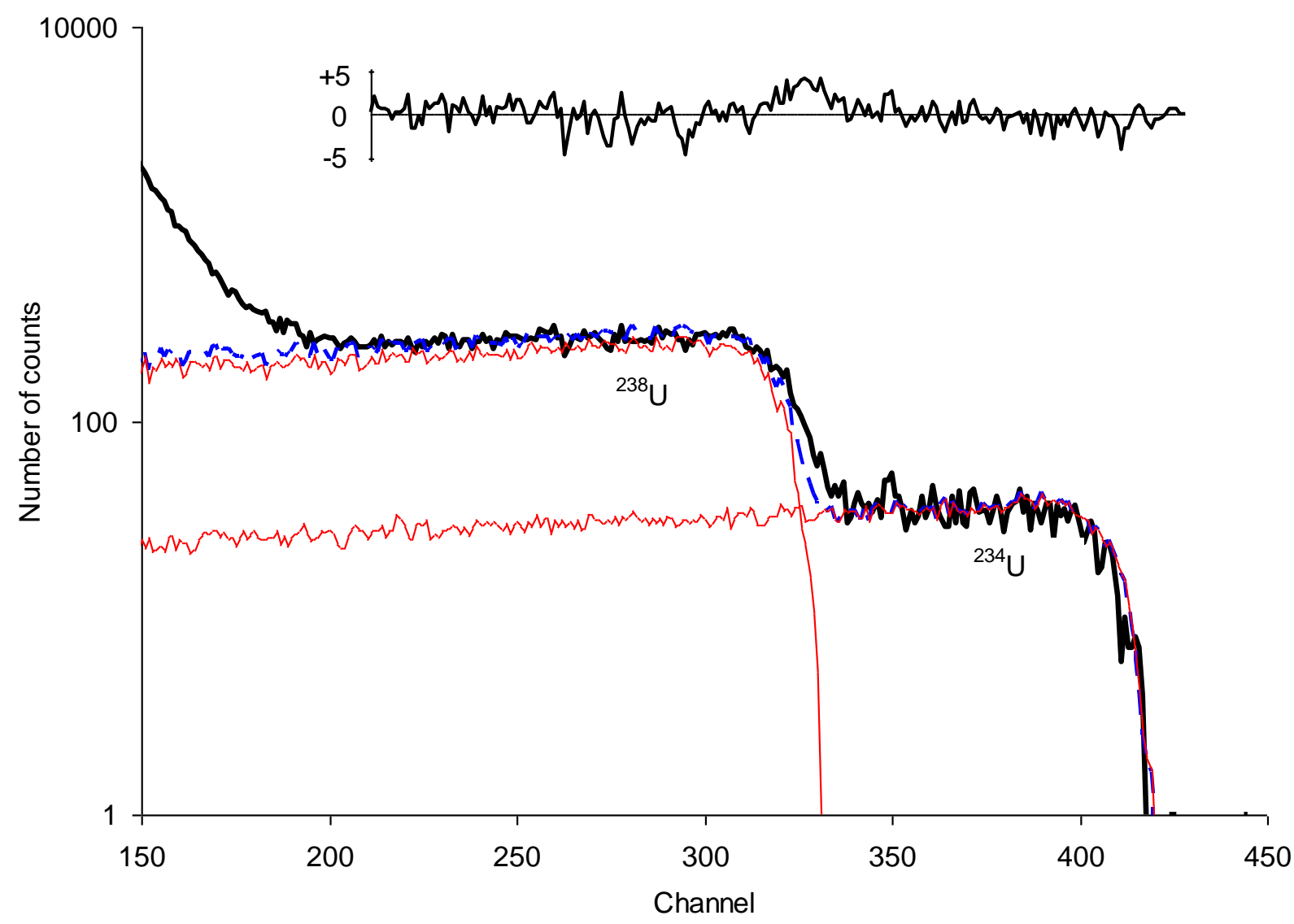

Fig. 3 Measured pulse height spectrum from the DU penetrator (thick black line), fitted peaks of ${ }^{234} U$ and ${ }^{238} U$ (thin red lines) and their sum (dashed blue line). The reduced residual of the fit is shown at top (in units of standard deviation of the number of counts). 
those which may be encountered in alpha spectrum measurements. Generally, these types of spectra can not be analyzed by commercial peak fitting programs with pre-described peak shapes.

The analysis of the spectrum starts with AASI simulations to obtain initial values of the source characteristics for the fitting. In this particular case, the simulations are used to examine which alpha-particle emitting nuclides are present in the penetrator and what are their activity ratios. To accomplish this, simulated alpha spectra for different candidate nuclides are generated. Those nuclides whose high-energy edge of the peak coincides with that of the measured spectrum are included in the fitting. As indicated in Fig. 3, the nuclides considered here are ${ }^{234} \mathrm{U}$ and ${ }^{238} \mathrm{U}$. This comprises the first step in the fitting. Because of the limited statistics in the measured spectrum the presence of minute quantities of e.g. ${ }^{235} \mathrm{U}$ and ${ }^{236} \mathrm{U}$ cannot be excluded in this analysis. However, the simulations and fitting can be used to estimate the largest possible amount of undetected alpha emitters that may be present in the measured source. The nuclide composition of the source is assumed to be homogeneous. Because of the large size of the source compared to the range of alpha particles, its density and dimensions have no influence on the fitting.

As soon as the nuclides are selected, the area fitting with AASIFIT can start to obtain activity ratio of the selected nuclides. Absolute activities cannot be estimated because of the source thickness. In the present case, there are no other parameters except the peak areas to be fitted. The region-of-interest is chosen so that the region below channel 210 is excluded because there the spectrum is dominated by the pulses from the beta decay electrons.

After the fit has converged, AASIFIT reports the fitted peak areas. The resulting areas are 11,200 for ${ }^{234} \mathrm{U}$ and 60,700 for ${ }^{238} \mathrm{U}$. Uncertainties in the fitted peak areas are $2.8 \%$ and $1.1 \%$, respectively. In the case of a thin source the activity ratio of ${ }^{234} \mathrm{U}$ to ${ }^{238} \mathrm{U}$ could be directly estimated from the peak areas. However, since a thick source is considered here, a slightly different approach is needed to account for the alpha particles totally absorbed by the penetrator: Since the initial alpha particle energies of these nuclides differ (4.8 MeV for ${ }^{234} \mathrm{U}$ vs. $4.2 \mathrm{MeV}$ for ${ }^{238} \mathrm{U}$ ), the alpha particles emitted by ${ }^{238} \mathrm{U}$ are, on average, emitted closer to the projectile surface than those emitted by ${ }^{234} \mathrm{U}$. For the activity ratio calculation equal volumes for these nuclides must be considered: here, this is accomplished by correcting the activity ratio with the ratio of projected alpha particle ranges in uranium. The resulting activity ratio, 0.15 , shows that the $U$ has been processed - unprocessed $U$ has the activity ratio close to 1 . We note that the analysis of Reference 10 resulted in activity ratio of 0.122 .

${ }^{235} \mathrm{U}$ and ${ }^{236} \mathrm{U}$ were not considered in the fit. However, the residual of the fit (Fig. 3) shows that there are no other major peaks present in the measured spectrum. Therefore the activities of ${ }^{235} \mathrm{U}$ and ${ }^{236} \mathrm{U}$, if present, are much smaller than those of ${ }^{234} \mathrm{U}$ and ${ }^{238} \mathrm{U}$. This finding is supported by the results of Reference 10 .

\section{Depth Distribution of Aerosol Particles in a Glassfibre Filter}

The alpha particle energy spectrum registered from an aerosol sample is used to illustrate the iterative fitting capabilities of AASIFIT. The sample was collected indoors on a glassfibre filter and the alpha particle energy spectrum was registered in a vacuum chamber. The aerosol particles, including the alpha-particle emitting radon progeny, penetrate into the filter. Thus, the alpha particles travel through the filter material before reaching the detector causing notable peak tailing in the measured spectrum (Fig. 4). The depth distribution of the aerosol particles is assumed to be exponential and, thus, the distribution can be characterized by a single parameter, the mean penetration depth.

In the simulations the aerosol size distribution was assumed to be log-normal with the mean diameter of $0.4 \mu \mathrm{m}$ and the geometric standard deviation of 1.3. AASIFIT was used to fit the measured spectrum by varying the mean penetration depth of the aerosol particles in the filter. The best chi-squared value was obtained when the mean penetration depth was $95 \mu \mathrm{m}$.

Figure 4 shows the result of the fit. The residual varies evenly about zero at the tails of the spectrum peaks and it can be concluded that the fit describes well the measured spectrum. The effect of varying penetration depth on the simulated spectrum is schematically illustrated by the shaded areas in Fig. 4. The larger the penetration depth, the more counts are seen at the tails of the peaks and vice versa.

\section{Discussion and Conclusion}

The AASI Monte Carlo program has been applied in analyses of environmental and safeguards samples, laboratory quality control and in analyses of complex alpha spectra with non-optimal peak shapes. The program is, in particular, designed to help the analysis of spectra obtained in non-destructive alpha spectrometry. Combined to AASI, a fitting program called AASIFIT is introduced to fit the simulated alpha spectrum to the measured one. In AASIFIT the Monte Carlo simulations and spectrum fitting are iterated to obtain a best possible match. The iteration process facilitates obtaining additional information on the source properties. For instance, in case of radioactive particles information on the particle density or shape/diameter can be obtained.

In this contribution, capabilities of the AASIFIT program are demonstrated using a DU penetrator found in Kosovo as an example. Simulations and fitting are used to quantify the alpha particle emitting nuclides present in the projectile. The same approach can be used to assess the relative amounts of alpha emitters in almost any microscopic or macroscopic sample - in particular suspected nuclear material sample that may contain $\mathrm{U}$ and $\mathrm{Pu}$. This quantification may be done in the field and without any radiochemical sample treatment. The method described in this work facilitates and speeds up the analyses in the case of a nuclear incident or in safety, security and safeguards applications.

To demonstrate the iterative fitting procedure, the mean penetration depth of the aerosol particles distributed in a 


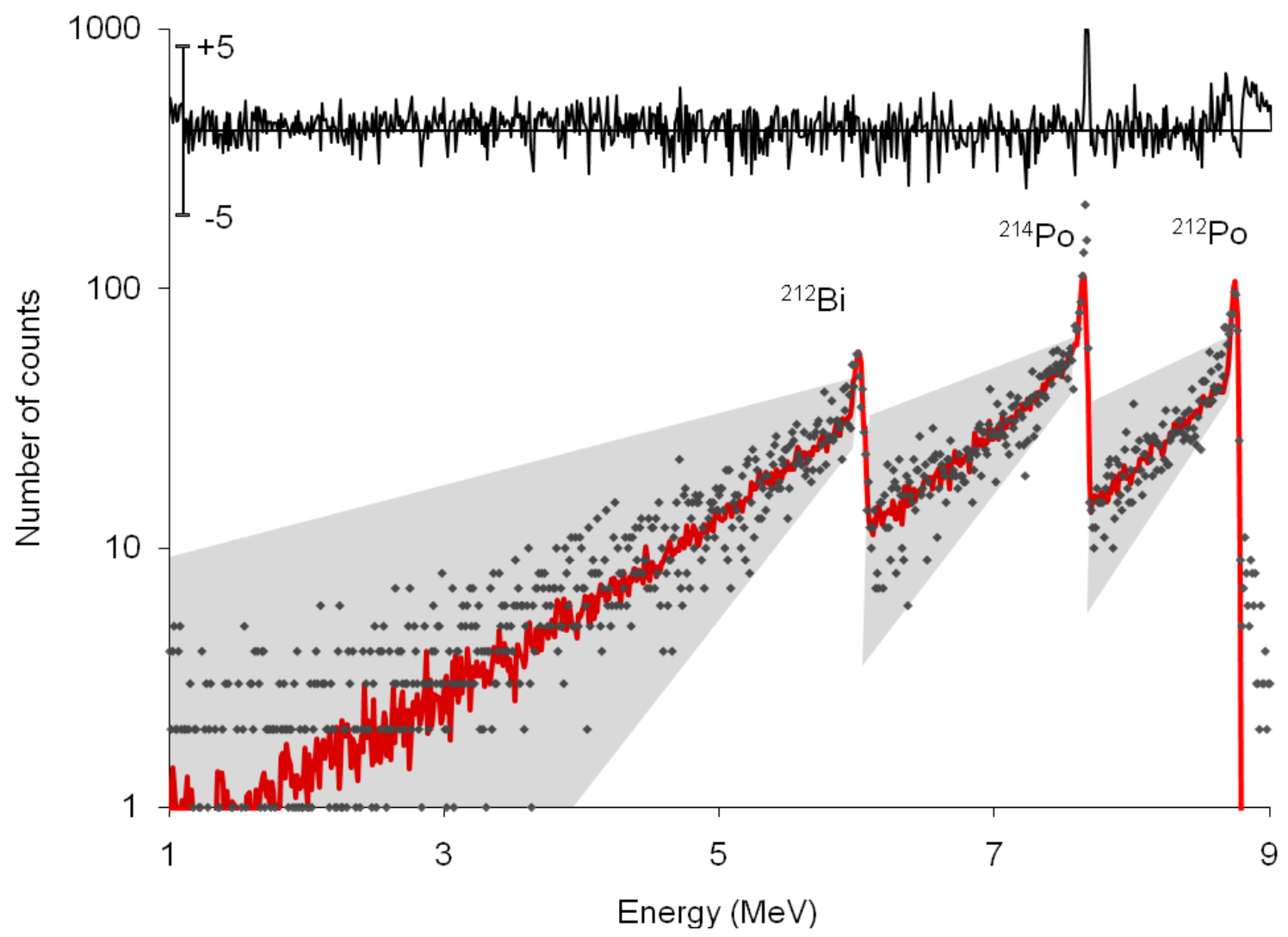

Fig. 4 Measured pulse height spectrum from the glassfibre filter (markers) and fitted peaks of ${ }^{212} \mathrm{Po},{ }^{214} \mathrm{Po}$ and ${ }^{212} \mathrm{Bi}$ (solid line). The reduced residual of the fit is shown at top (in units of standard deviation of the number of counts). The shaded areas illustrate the effect of varying mean penetration depth on the simulated spectrum.

glassfibre filter is examined.

The AASI program is freely available for download at http://www.stuk.fi/tutkimus/programs/aasi/en_GB/aasi/.

\section{References}

1) Proceedings of an international conference on "Advances in destructive and non-destructive analysis for environmental monitoring and nuclear forensics", Karlsruhe, Germany, 21-23 October 2002.

2) K. Mayer, M. Wallenius, I. Ray, "Tracing the origin of diverted or stolen nuclear material through nuclear forensic investigations", pp. 389-408, In: R. Avenhaus, N. Kyriakopoulos, M. Richard, G. Stein (editors), Verifying Treaty Compliance, Springer, Berlin-Heidelberg (2006).

3) T. Siiskonen, R. Pöllänen, "Advanced simulation code for alpha spectrometry", Nucl. Instr. Meth. Phys. Res., A550, 425-434 (2005).

4) R. Pöllänen, T. Siiskonen, "Direct alpha spectrometry from swipe samples", In: Proceedings of the 29th ESARDA Annual Meeting, May 22-24 (2007), Aix en Provence, France.
5) Y. Ranebo, R. Pöllänen, M. Eriksson, T. Siiskonen, N. Niagolova, "Characterization of radioactive particles using non-destructive alpha spectrometry", Appl. Radiat. Isot. 68, 1754-1759 (2010).

6) R. Pöllänen, T. Karhunen, T. Siiskonen, H. Toivonen, A. Pelikan, "Deconvolution of alpha spectra from hot particles", In: Oughton, D., Kashparov, V. (eds), Radioactive Particles in the Environment, NATO Science for Peace and Security Series C: Environmental Security, Springer, 209-220 (2009).

7) T. Siiskonen, R. Pöllänen, T. Karhunen, "A versatile simulation code for alpha spectrometry: Development of the graphical user interface and applications", ESARDA bulletin, 40, 26-30 (2008).

8) K. Levenberg, "A Method for the Solution of Certain Non-Linear Problems in Least Squares", The Quarterly of Applied Mathematics, 2, 164-168 (1944).

9) D. Marquardt, "An Algorithm for Least-Squares Estimation of Nonlinear Parameters". SIAM Journal on Applied Mathematics, 11, 431-441 (1963).

10) R. Pöllänen et al., "Characterisation of projectiles composed of depleted uranium", J. Environ. Radioactivity, 64, 133-142 (2003). 